OPEN ACCESS

Edited by:

Sofia A. Casares,

Naval Medical Research Center.

United States

Reviewed by:

Irina V. Kiseleva,

Institute of Experimental Medicine

(RAS), Russia

Wayne Robert Thomas,

University of Western Australia,

Australia

*Correspondence:

Molalegne Bitew

molalegne23@gmail.com

Specialty section:

This article was submitted to Vaccines and Molecular Therapeutics,

a section of the journal

Frontiers in Immunology

Received: 01 September 2021 Accepted: 02 November 2021

Published: 30 November 2021

Citation:

Dessalegn B, Bitew M, Asfaw D, Khojaly E, Ibrahim SM, Abayneh $T$,

Gelaye $E$, Unger $H$ and Wijewardana $V$

(2021) Gamma-Irradiated Fow

Cholera Mucosal Vaccine: Potential

Vaccine Candidate for Safe and

Effective Immunization of

Chicken Against Fowl Cholera.

Front. Immunol. 12:768820.

doi: 10.3389/fimmu.2021.768820

\section{Gamma-Irradiated Fowl Cholera Mucosal Vaccine: Potential Vaccine Candidate for Safe and Effective Immunization of Chicken Against Fowl Cholera}

\author{
Bereket Dessalegn ${ }^{1}$, Molalegne Bitew ${ }^{2 *}$, Destaw Asfaw ${ }^{1}$, Esraa Khojaly ${ }^{3}$, \\ Saddam Mohammed Ibrahim ${ }^{1}$, Takele Abayneh ${ }^{4}$, Esayas Gelaye ${ }^{4}$, Hermann Unger ${ }^{5}$ \\ and Viskam Wijewardana ${ }^{5}$
}

\footnotetext{
${ }^{1}$ College of Veterinary Medicine and Animal Science, University of Gondar, Gondar, Ethiopia, ${ }^{2}$ Health Biotechnology Directorate, Ethiopian Biotechnology Institute, Addis Ababa, Ethiopia, ${ }^{3}$ MSc Program on Vaccine Production and Quality Control, Pan Africa University for Life and Earth Sciences Institute (PAULESI), University of Ibadan, Ibadan, Nigeria, ${ }^{4}$ Vaccine Research and Development Directorate, National Veterinary Institute, Debre Zeit, Ethiopia, ${ }^{5}$ Animal Production and Health Section, Joint Food and Agriculture Organization (FAO)/International Atomic Energy Agency (IAEA) Centre of Nuclear Techniques in Food and Agriculture, International Atomic Energy Agency (IAEA), Vienna, Austria
}

Fowl cholera (FC) caused by Pasteurella multocida is among the serious infectious diseases of poultry. Currently, formalin inactivated FC (FI-FC) vaccine is widely used in Ethiopia. However, reports of the disease complaint remain higher despite the use of the vaccine. The aim of this study was to develop and evaluate gamma-irradiated mucosal FC vaccines that can be used nationally. In a vaccination-challenge experiment, the performance of gamma-irradiated $P$. multocida (at $1 \mathrm{kGy}$ ) formulated with Montanide gel/01 PR adjuvant was evaluated at different dose rates $(0.5$ and $0.3 \mathrm{ml})$ and routes (intranasal, intraocular, and oral), in comparison with $\mathrm{Fl}-\mathrm{FC}$ vaccine in chicken. Chickens received three doses of the candidate vaccine at 3-week intervals. Sera, and trachea and crop lavage were collected to assess the antibody levels using indirect and sandwich ELISAs, respectively. Challenge exposure was conducted by inoculation at $3.5 \times 10^{9} \mathrm{CFU} /$ $\mathrm{ml}$ of $P$. multocida biotype A intranasally 2 weeks after the last immunization. Repeated measures ANOVA test and Kaplan Meier curve analysis were used to examine for statistical significance of antibody titers and survival analysis, respectively. Sera IgG and secretory IgA titers were significantly raised after second immunization ( $D=0.0001$ ). Chicken survival analysis showed that intranasal and intraocular administration of the candidate vaccine at the dose of $0.3 \mathrm{ml}$ resulted in 100\% protection as compared to intramuscular injection of $\mathrm{FI}-\mathrm{FC}$ vaccine, which conferred $85 \%$ protection ( $p=0.002)$. In conclusion, the results of this study showed that gamma-irradiated FC mucosal vaccine is safe and protective, indicating its potential use for immunization of chicken against FC.

Keywords: chicken, fowl cholera, gamma radiation, mucosal vaccine, P. multocida 


\section{INTRODUCTION}

Poultry production contributes significantly to the livelihoods of farmers and to the national economic system. However, it is hampered by various factors, including poor husbandry practices and poultry diseases (1). Fowl cholera (FC), which is caused by $P$. multocida, is among the serious infectious diseases of poultry. The disease is present globally and endemic to most parts of Ethiopia with significant economic losses associated with reduced production and mortality (2). It is vital and preferable to develop vaccines from locally circulating strains to provide a robust protection (3). Despite the contribution by the locally produced formalin-killed FC vaccine in reducing the disease burden, FC remains to be a big challenge to the poultry sector in the country. This in part can be explained by the fact that the current used formalin-inactivated FC vaccine produced by the National Veterinary Institute (NVI) confers a short duration of protection (4). In addition, owing to its nature of being inactivated parenteral preparation, the vaccine is expected to be a poor inducer of mucosal immunity, which is the desired protective immunity against mucosal pathogens such as avian Pasteurella (5).

The route of vaccine administration plays an important and significant role in practical usage. The fact that parenteral vaccines induce little-to-none mucosal immunity makes them poor and ineffective choice to immunize against mucosal pathogens (6). In addition, mucosal vaccines are easy to administer and is preferable in case of vaccination campaigns and in farm settings where there is large number of chickens to be vaccinated (7). In general, parenteral preparations induce short-lived humoral immunity, which necessitates booster doses. However, mucosal vaccines elicit long-lived immunity of both humoral and cellular nature (8). Therefore, there is an urgent need to develop effective and safe mucosal vaccines that confer long duration of protection against mucosal pathogens.

The commonly used chemical inactivation methods has limitations associated with safety (probably correlated with the high endotoxin level) and efficacy due to modification of antigenic components of bacteria, making it less immunogenic and potent. Furthermore, these vaccine antigens are mostly presented through major histocompatibility complex (MHC)-II but not MHC-I pathways by antigen-presenting cells and do not result in an efficient cell-mediated immune response that is crucial against many pathogens. Considering recombinant method of vaccine development against FC might not be a feasible approach for developing countries because they need high technical expertise, high-technical facilities, and resource limitations, as well as the immunity conferred is very limited and narrow (9).

Radiation inactivation of pathogens has potential applications in sterilization and the manufacture of biological reagents and laboratory supplies (10). Exposure to optimum doses of gamma radiation disrupts the genetic material of the pathogens, making the microorganism unable to replicate, so it cannot establish an infection yet leaving some residual metabolic activity. Therefore, the irradiated microorganisms may still find its natural target in the host and could effectively be immunogenic (11). The major advantage of ionizing radiation in vaccine development compared to ultraviolet or chemical agents is its ability to effectively penetrate through most biological materials and specifically target nucleic acids whilst causing less damage to surface antigenic protein, making it preferable to develop safe and simple vaccines (12). Gamma-irradiated vaccines appear to be more effective than formalin-killed vaccines against disease and have the added advantage of a longer storage life than live vaccines (13). Therefore, the present study was aimed to develop an improved gamma-irradiated inactivated vaccine against fowl cholera that stimulates the enhanced mucosal immune response and is easy for application at the rural setting.

\section{MATERIALS AND METHODS}

\section{Study Site}

The study was conducted at the National Veterinary Institute (NVI), Bishoftu; National Institute for Control and Eradication of Tsetse Fly and Trypanosomosis (NICETT), Addis Ababa; and Ethiopian Biotechnology Institute, Addis Ababa, from November 2020 to June 2021.

\section{Experimental Chicken and Their Management}

In this experiment, 250 3-week-old specific antibody negative (SAN) against FC Bovans brown chickens were used. The parent stock was not vaccinated against FC. Chicks used for all the experiments were raised under intensive management system. The animal experiment rooms were cleaned with disinfectants and fumigated with formalin before the introduction of chicks and bedded with disinfected wood shavings. The chickens had access to feed and water ad libitum throughout the experiment.

\section{Preparation of Avian P. multocida Inoculum: For Preliminary Study, Vaccine Preparation, and the Challenge Study}

Working seeds of Avian P. multocida biotype A (MK802880, NVI) were used for vaccine preparation. Lyophilized $P$. multocida biotype A was diluted with $2 \mathrm{ml}$ of tryptose soya broth (TSB), homogenized well and then inoculated into sterile tryptose soya agar (TSA) supplemented with $10 \%$ horse serum and incubated at $37^{\circ} \mathrm{C}$ overnight. The identity of this isolate was confirmed by both phenotypic and molecular standard tests. A single colony was transferred to $2 \mathrm{ml}$ tube containing TSB with $10 \%$ horse serum and incubated for $7 \mathrm{~h}$ at $37^{\circ} \mathrm{C}$. Then $0.5 \mathrm{ml}$ of the broth culture was transferred into $30 \mathrm{ml}$ TSB supplemented with $10 \%$ horse serum and incubated overnight. The purity of the P. multocida type A (PA) inoculum was checked and inoculated into PA production media at the ratio of $7 \mathrm{ml}$ of inoculum, $7 \mathrm{ml}$ of glucose, and $3 \mathrm{ml}$ of serum per $300 \mathrm{ml}$ of $P$. multocida production media, then incubated for $24 \mathrm{~h}$ with slow agitation at $80 \mathrm{rpm}$ (14). The culture was harvested at the $\mathrm{pH}$ of 5.5 to 6.2 , which is known to correspond to the desired titer of $10^{9} \mathrm{CFU} / \mathrm{ml}$ and above as determined by the plate count method. In addition, avian $P$. multocida strain was used as challenge strains in the test of 
the vaccines. Freeze-dried stock was reconstituted with $2 \mathrm{ml}$ tryptose broth (TB), and suspensions were streaked on tryptose soya agar (TSA) plates incubated for $24 \mathrm{~h}$ at $37^{\circ} \mathrm{C}$. The culture was checked for purity and identity. From the culture on TSA, a typical colony was inoculated to $200 \mathrm{ml}$ TSB and incubated for $7 \mathrm{~h}$ at $37^{\circ} \mathrm{C}$. These cultures were then adjusted spectrophotometrically at $450 \mathrm{~nm}$ ( 0.475 OD value) and serially diluted in TSB to obtain the desired titer for challenge $\left(3.5 \times 10^{9} \mathrm{CFU} / \mathrm{ml}\right)$.

\section{Determination of Appropriate Gamma Radiation Dose for Optimum Inactivation of Avian P. multocida}

The PA production media containing culture was centrifuged at $4,000 \times \mathrm{g}$ per minute at $4^{\circ} \mathrm{C}$ for $20 \mathrm{~min}$ after determining the time required to obtain the desired titer $\left(5.6 \times 10^{9} \mathrm{CFU} / \mathrm{ml}\right)$. The supernatant was discarded, and the cell pellet was washed twice with PBS and resuspended in PBS with equal volume in falcon tubes and subjected to gamma irradiation with doses ranging from 0.5 to $3 \mathrm{kGy}$ at a dose rate of $1.56 \mathrm{kGy} / \mathrm{h}$ using a cobalt 60 irradiation machine (MDS NORDION, Canada) $(15,16)$.

The falcon tubes containing the culture were placed vertically and securely in the gamma chamber and irradiated for different time periods according to the required doses of gamma rays. The temperature inside the gamma chamber was maintained at 37$40^{\circ} \mathrm{C}$. After completion of irradiation, each tube was carefully removed from the gamma chamber and immediately stored at $-4^{\circ} \mathrm{C}$ for further use. Non-irradiated controls underwent the same procedure except irradiation. The facility at the National Institute for Control and Eradication of Tsetse Fly and Trypanosomosis (NICETT) at Addis Ababa was utilized for this purpose. Bactericidal activity of the radiation dose was assessed by subculturing of serial dilution of $P$. multocida cells plated on tryptose soya agar plates to quantify CFU. Various irradiation doses were examined to find the lowest optimum irradiation at the margin of the lethal dose (17).

\section{Safety and Immunogenicity Study of Avian $P$. multocida Irradiated at Different Dose of Gamma Radiation}

Avian P. multocida preparations irradiated at four consecutive irradiation doses close to complete lethal dose and adjuvanted with $20 \%$ of Montanide/01 PR gel adjuvant were evaluated for their immunogenicity and safety. The inoculum preparation of avian $P$. multocida used for challenge was done as indicated in above. Thirty chickens were randomly divided into five groups with six chickens in each group and were intranasally inoculated with $1 \mathrm{ml}$ of candidate mucosal FC vaccine irradiated with 0.9 kGy (group 1), candidate mucosal FC vaccine irradiated with 1 kGy (group 2), candidate mucosal FC vaccine irradiated with $1.1 \mathrm{kGy}$ (group 3), candidate mucosal FC vaccine irradiated with $1.2 \mathrm{kGy}$ (group 4), and control inoculated with PBS (group 5). Following vaccination, chickens were monitored daily for any behavioral changes. Blood samples were collected from the wing vein at days 0,14 , and 21 postvaccination to determine the antibody titer raised against avian
P. multocida biotype A (PA) using the indirect ELISA (Product code: PMS-CHICK-5P, IDvet, France). Safety was assessed by monitoring administration site reactions such as pain and swelling, systemic reactions like fever and anorexia, and lesion in the liver and spleen.

\section{Formulation of the Candidate Gamma- Irradiated Mucosal FC Vaccine}

The 1 kGy gamma-irradiated avian Pasteurella multocida was chosen for the vaccine preparation since it performed best in antibody production using I-ELISA test. The inoculum preparation of avian $P$. multocida was done as mentioned in section 3.2.1. The irradiated culture of avian $P$. multocida $\left(5.6 \times 10^{9} \mathrm{CFU} / \mathrm{ml}\right)$ was adjuvanted with Montanide/01 PR gel to form the final vaccine preparation. The proportion of Montanide/01 PR gel adjuvant is made to comprise $20 \%$ of the antigen preparation as recommended by the adjuvant manufacturer (18). Then, the vaccine was dispensed into vials of $50 \mathrm{ml}$ volume capacity and checked for its purity and sterility by using Gram's stain and culturing on sterility test media such as tryptose agar, tryptose broth, and Sabouraud agar media. Finally, the gamma-irradiated fowl cholera vaccine was found free from any contamination.

\section{Evaluation of the Final Candidate Vaccine}

Chickens were allocated into seven groups, G-1 to G-5 based on the dose of candidate mucosal FC vaccine they received and the route of administration. Thirty-six (36) chickens from both G-1 and G-2 received the vaccine intranasally (IN) at a dose of 0.5 and $0.3 \mathrm{ml}$, respectively. Similarly, 36 chickens from G-3 received the vaccine at a dose of $0.5 \mathrm{ml}$ orally. On the other hand, 20 chickens both from G-4 and G-5 were administered with 0.5 and $0.3 \mathrm{ml}$ of the vaccine intraocularly (IO), respectively. All the chickens (G-1 to G-5) received three doses of the vaccine preparations at 3-week interval. Another two groups of chickens, G-6 (36 chickens) and G-7 (36 chickens), were used as a comparator and placebo control, respectively. Chickens in G-6 were administered three doses of $0.5 \mathrm{ml}$ of the commercial formalin-inactivated FC vaccine $(1 / 20)$ at 3 -week interval intramuscularly. Finally, all chickens from G-7 received PBS (Figure 1).

\section{Assessment of the Safety of Mucosal FC Vaccine}

Evaluation of the safety of the vaccine was done according to the OIE manual for vaccine safety parameter (3), vaccinated chickens were observed the whole day starting from the time of vaccination up to the end of the experiment on a daily basis, and any deviation from normal health using observation of vital signs was recorded: depression, anorexia, ruffled feather, and any reaction at the site of injection.

\section{Assessment of Serum and Mucosal Antibody Response}

Antibody responses, serum IgG, and secretory IgA in chickens were determined by ELISA. Blood samples were collected on days 0, 14, 


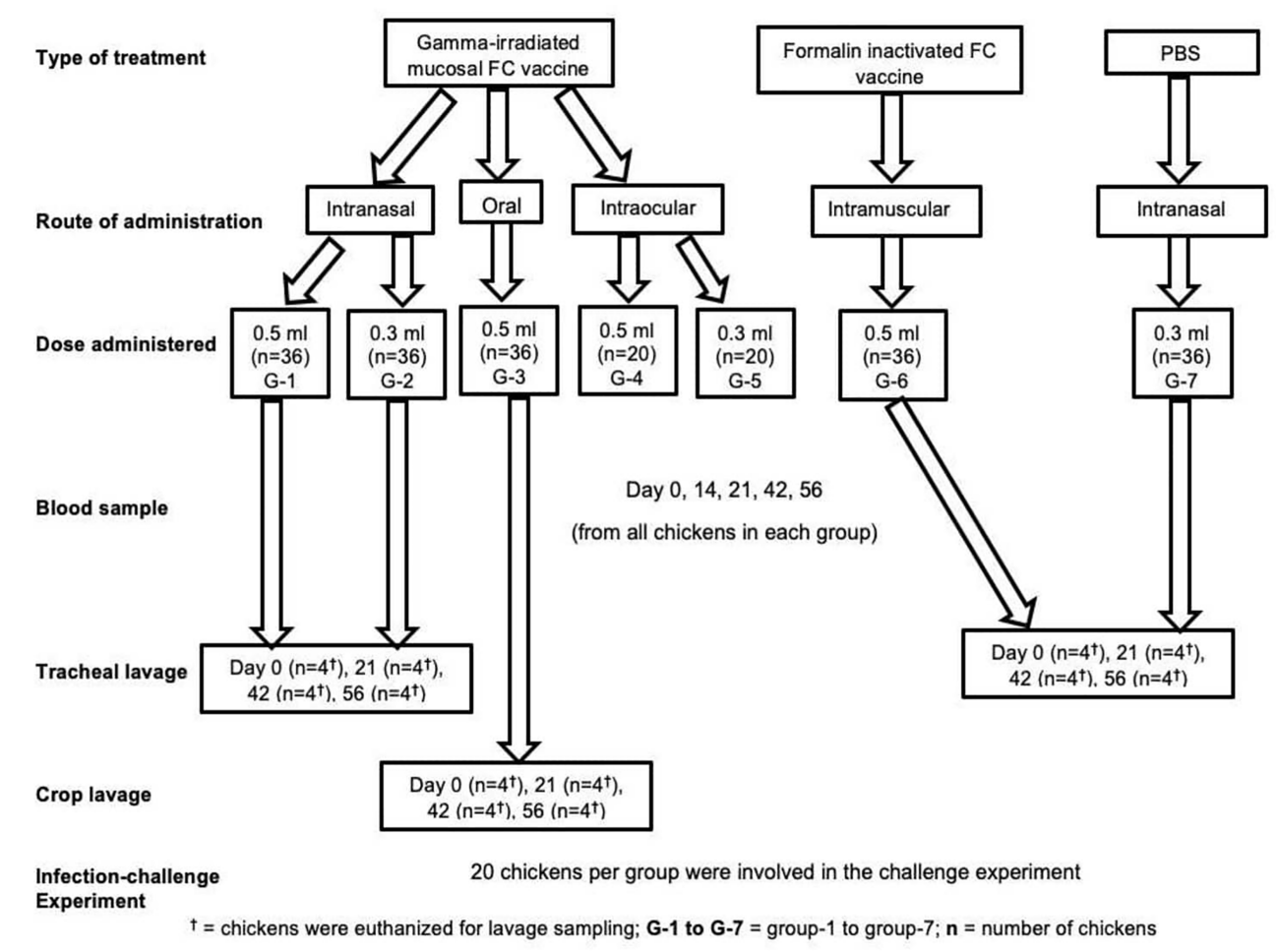

FIGURE 1 | Experimental design groups; treatment, comparator, and control, route of vaccine administration, and number of chickens in each group, number of chickens used in sampling, and number of chickens used in efficacy study.

21,42 , and 56 of the experiments. In addition, four chickens in each group except intraocular route were sampled and euthanized on days $0,21,42$, and 56 of the experiments, and the tracheal and crop lavage was performed (Figure 1). Then, the sera or tracheal and crop lavage solutions were subjected to ELISA procedures. Antibody responses in the chicken sera were determined by measuring of the IgG titers using a commercial indirect ELISA test kit (Product code: PMS-CHICK-5P, IDvet, France). In addition, the secretory IgA was measured using a chicken IgA sandwich ELISA Kit (CAT. No: MBS564152MyBioSource, San Diego, USA). The average antibody titer and the standard error of the mean (SEM) of each group were computed according to the company's recommendation.

\section{Assessment of Efficacy of the Candidate Vaccine}

As indicated in Figure 1, 20 chickens from all groups were challenged with avian P. multocida at a dose of $3.5 \times 10^{9} \mathrm{CFU} / \mathrm{ml}$ 2 weeks after the final vaccination. Preparation of avian $P$. multocida for challenge study was done as indicated in the above section. The chickens were followed up for clinical signs and mortality for 14 days. Necropsy and bacterial isolation were conducted on dead chickens. The gross lesions were recorded, and lungs, livers, and spleens were collected for bacterial isolation by direct culture using TSA with $10 \%$ horse serum followed by identification through morphology, staining, culture, and finally by species-specific PCR.

\section{Data Analysis}

GraphPad Prism 9 was used to perform statistical analysis. The antibody titers between the vaccinated groups and the nonvaccinated control group were performed using a repeated measures ANOVA test and Tukey multiple comparisons. The level of significance was recorded at $\mathrm{p}<0.05$. The data were presented as individual values for each experimental group. Mean and standard error of means are indicated in lines and error bars. The survival of chickens was compared between different treatment and in vivo infection challenge groups using Kaplan-Meier curve analysis.

\section{RESULTS}

\section{Effects of Gamma Irradiation on Avian P. multocida}

Irradiation experiments were conducted to determine the dose required for the inactivation of avian $P$. multocida. An exponential decrease in viability of avian P. multocida was observed while increasing the dose of gamma irradiation. After $48 \mathrm{~h}$ of culturing, avian $P$. multocida exposed to doses more than or equivalent to 1 $\mathrm{kGy}$ of irradiation had fully inhibited replication, and no growth was seen (Figure 2). The susceptibility of surface structural proteins to reactive oxygen species (ROS) damage increases when the radiation dose is higher than the level that completely 


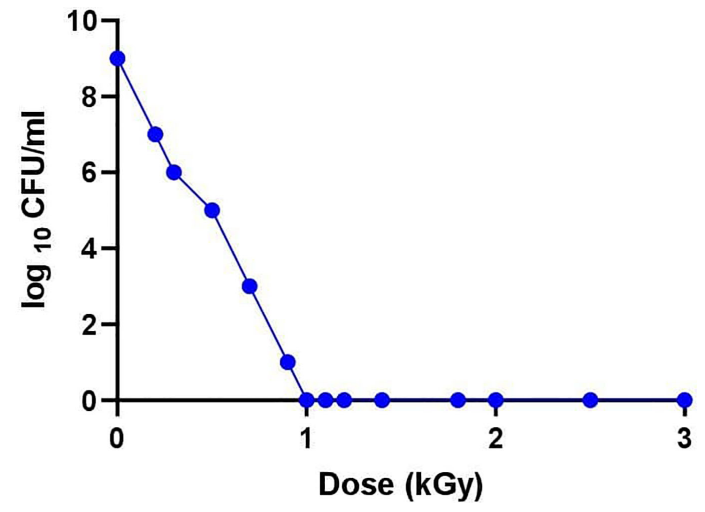

FIGURE 2 | After determination of the desired titer (5.6×109 CFU/ml), the broth culture was centrifuged at 4,000 revolutions per minute at $4^{\circ} \mathrm{C}$ for 20 min. The supernatant was discarded, and the pellet of cells was washed twice with PBS and resuspended in $5 \mathrm{ml}$ PBS in $15 \mathrm{ml}$ falcon tubes and subjected to gamma irradiation with doses ranging from 0 to $3 \mathrm{kGy}$ at a dose rate of $1.56 \mathrm{kGy} / \mathrm{h}$ using a cobalt 60 irradiation machine (MDS NORDION, Canada). Bactericidal activity of the radiation dose was assessed by subcultivation of serial dilution of $P$. multocida cells plated on tryptose soya agar plates to quantify CFU. Various irradiation doses were examined to find the lowest optimum irradiation at the margin of the lethal dose.

abolished avian P. multocida. Therefore, the lethal dose (1 kGy) and three doses close to the lethal dose $(0.9,1.1,1.2 \mathrm{kGy})$ were used to select immunogenic dose and safety of irradiated avian $P$. multocida in chicken.

\section{Immunogenicity and Safety of Radiation Inactivated Avian P. multocida Vaccine Preparations}

The safety and immunogenicity of several vaccine preparations made from avian $P$. multocida treated with various doses of gamma radiation adjuvanted with Montanide/01 PR gel (SEPPIC, France) was investigated. Briefly, 30 chickens were assigned into five groups (six chickens per group); four groups were administered $5.6 \times 10^{9} \mathrm{CFU} / \mathrm{ml}$ of avian $P$. multocida irradiated with $0.9,1,1.1$, and $1.2 \mathrm{kGy}$ of gamma ray formulated with Montanide/01 PR gel through IN route. Another group of six chickens received PBS and was used as a control. At 14 and 21 days after vaccination, significantly higher levels of PA-specific IgG antibodies were identified in the serum of the four vaccinated groups when compared to the PBSinoculated control groups (Figure 3). In chickens immunized with irradiated avian $P$. multocida at $1 \mathrm{kGy}$, the average antibody titer was $0.345 \pm 0.095$ on day 14 and $0.43 \pm 0.12$ on day 21 , while in chickens immunized with $0.9 \mathrm{kGy}$ irradiated was $0.22 \pm 0.06$ and $0.31 \pm 0.11$. On the other hand, chickens immunized with 1.1 and $1.2 \mathrm{kGy}$ irradiated avian P. multocida produced similar average antibody titer $0.21 \pm 0.06$ on day 14 and $0.26 \pm 0.11$ on day 21 . When compared to the other vaccinated chickens (different gamma irradiation doses), chickens vaccinated with 1 kGy gamma irradiation candidate vaccine generated significantly higher levels of IgG antibodies in the serum at days 14 and 21 post-vaccination $(p=0.0001)$. Furthermore, chickens vaccinated with $0.9 \mathrm{kGy}$ gamma-irradiated candidate vaccine had higher levels of antibodies as compared to the remaining groups $(p=0.04)$. There was no clinical evidence of sickness or injection site reactions in any of the vaccinated chickens. In all vaccinated groups, no lesion was found in the liver or spleen of chickens. Irradiation at $1 \mathrm{kGy}$ was chosen as optimum irradiation dose based on immunogenicity and safety data.

\section{Evaluation of Serum and Mucosal Antibody Response Against Mucosal FC Vaccine}

Determination of serum IgG titers using an indirect ELISA is shown in Figure 4. The levels of antibody titers of chickens against FC on day 0 indicated a low cutoff value of 0.2 . The levels of chicken serum IgG titers were found to be significantly increased after 2 weeks of the first immunization with the gamma-irradiated or formalin-inactivated vaccine. At the third week, a significant difference was observed among all vaccinated groups, where the average antibody titer of G-2 was $1.13 \pm 0.16$ compared to $0.97 \pm 0.18,0.59 \pm 0.096,0.61 \pm 0.08,0.77 \pm 0.17$, and $0.65 \pm 0.15$ for G-1, G-3, G-4, G-5, and G-6, respectively. After the second vaccination, the titers substantially increased in all chickens and were still significantly higher in the vaccinated chickens of gamma-irradiated mucosal FC vaccine than in the corresponding formalin-inactivated vaccinated group $(p=0.037)$ (Figure 4). Furthermore, peak of average antibody titer in G-1, G-2, and G-5 was observed after the second vaccination, which is $1.2 \pm 0.18,1.58 \pm 0.29$, and $1.26 \pm 0.24$, respectively. As compared to the other gamma-irradiated FC vaccinated groups, the average antibody titer of group-3 $(0.83 \pm 0,23)$ was low and showed significant difference compared to Group-2 $(p<0.009)$. However, there was no significant difference between the mean average antibody titer of groups of chickens vaccinated orally with the irradiated FC vaccine (G-3) and those vaccinated with commercial formalin-inactivated FC vaccine (G-6) throughout the experiment. After the third vaccination dose, the results of mean antibody titer of chickens in all groups were similar with second vaccination dose. On the other hand, the non-immunized group was found to be seronegative to FC, as the average antibody levels throughout the experimental period was lower than the cutoff value. Generally, the results indicated that the gamma-irradiated vaccine formulations are able to induce serum IgG against avian $P$. multocida. With regard to the route of vaccine administration, the average antibody titer levels of group $2(0.3 \mathrm{ml}$, intranasal route) generated significantly higher levels of serum IgG throughout the experiment ( $p=0.001)$. In addition, group-5 (0.3 ml, intraocular route) produced significantly higher antibody (IgG) titer after booster immunization $(p=0.001)$.

Secretory IgA was also detected in chickens immunized with the candidate gamma-irradiated mucosal fowl cholera vaccine (Figure 5). The levels of average IgA titers of chickens against FC on day 0 indicated a low cutoff value of 0.043 . At the third week, a significant difference was observed among chickens vaccinated with the gamma-irradiated vaccine intranasally at two dose rates, where the average IgA titer of G-1 and G-2 was $0.32 \pm 0.05$ and 


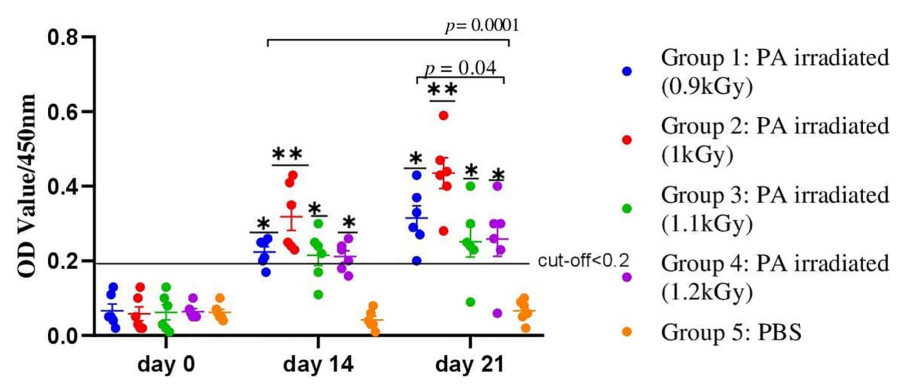

FIGURE 3 | A total of 30 chickens were assigned into five groups, with each group having six chickens. Chickens in groups $1,2,3$, and 4 received $5.6 \times 10^{9}$ CFU/ml of avian P. multocida irradiated with $0.9,1,1.1$, and 1.2 kGy of gamma rays and adjuvanted with Montanide/01 PR gel, respectively, through IN route. Chickens in group 5 received PBS and were used as a control. Blood samples were taken at days 0, 14, and 21. Serum was analyzed for the presence of lgG. The data were presented as individual values for each experimental group. Mean and standard error of means are indicated in lines and error bars. Asterisk $\left({ }^{\star}\right)$ represents the significant differentiation of antibody lgG level compared to the non-vaccinated control group $\left({ }^{*} p<0.05,{ }^{* *} p<0.01\right)$.

$0.36 \pm 0.12$ compared to the control group $(0.036 \pm 0.02)$. After the second vaccination, the titers substantially increased in all chickens. Comparative evaluation with formalin-inactivated FC vaccine showed that chickens vaccinated with the gammairradiated candidate vaccine displayed significantly higher average antibody titer of $1.23 \pm 0.06$ and $1.46 \pm 0.22$ in G-1 and G-2, respectively, than the chickens immunized with formalin-inactivated vaccine with $0.46 \pm 0.09$ mean value. After the third vaccination dose, a significant difference was observed among all vaccinated groups compared to the control group $(p<0.05)$. Like that of the serum IgG of chickens, the average IgA titers of the chickens vaccinated with gammairradiated FC vaccine orally were low and significantly different compared to the intranasal route $(p=0.026)$. In the control groups, no response was observed in antibody titers to avian P. multocida throughout the experiment. Generally, significant levels of IgA were detected only in gamma-irradiated mucosal fowl cholera immunized chickens, but not in that of formalininactivated fowl cholera immunized chickens $(p=0.034)$. These results suggest that gamma-irradiated mucosal fowl cholera vaccine is more potent in enhancing or inducing avian $P$. multocida-specific antibodies on the airway mucosal surface more than formalin-inactivated fowl cholera vaccine.

\section{Evaluation of Protective Efficacy of Mucosal FC Vaccine}

A total volume $0.5 \mathrm{ml}$ of bacterial suspension containing $3.5 \times 10^{9} \mathrm{CFU} / \mathrm{ml}$ of avian P. multocida biotype A was

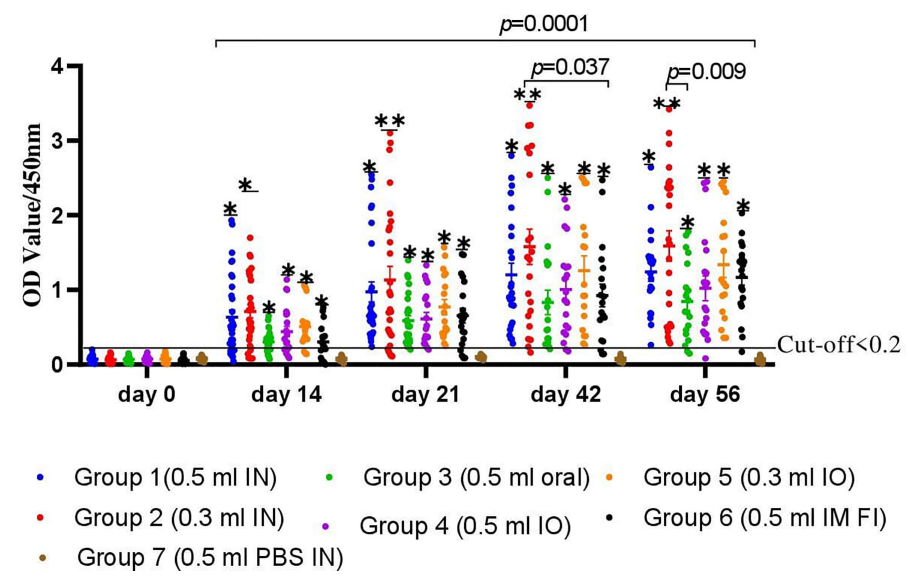

FIGURE 4 | A total of 220 chickens were assigned into seven groups: G-1 to G-5 based on the dose of candidate gamma-irradiated FC vaccine they received and the route of administration. Chickens in both $\mathrm{G}-1$ and $\mathrm{G}-2$ received the vaccine by intranasal route at a dose of 0.5 and 0.3 ml, respectively. Similarly, chickens in G3 received the vaccine at a dose of $0.5 \mathrm{ml}$ orally. On the other hand, G-4 and G-5 were administered 0.5 and $0.3 \mathrm{ml}$ of the vaccine through the intraocular route (IO), respectively. Another two groups of chickens, G- 6 and G-7, received $0.5 \mathrm{ml}$ of the commercial formalin-inactivated FC vaccine intramuscularly and $0.3 \mathrm{ml}$ of PBS intranasally as comparator, respectively. Blood samples were taken at days $0,14,21,42$, and 56 . Serum was analyzed for the presence of IgG. The data were presented as individual values for each experimental group. Mean and standard error of means are indicated in lines and error bars. Asterisk ( ${ }^{*}$ ) represents the significant differentiation of antibody lgG level compared to the non-vaccinated control group $\left({ }^{*} p<0.05,{ }^{* *} p<0.01\right)$. 


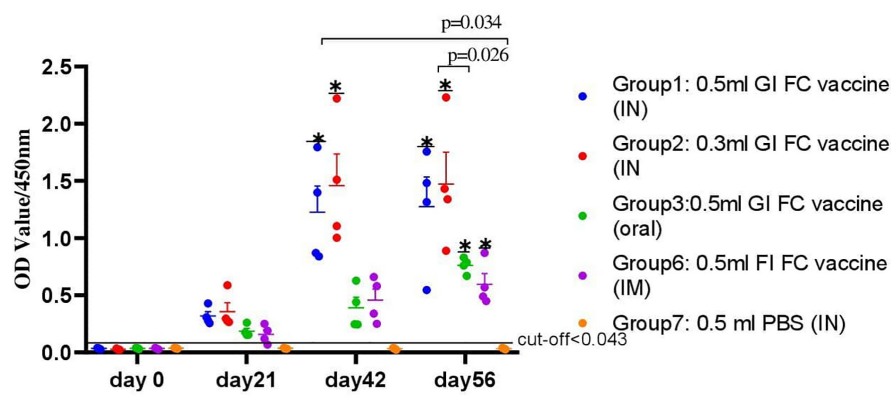

FIGURE 5 | A total of 80 chickens were chosen from all groups. Tracheal (G-1, G-2, G-4, and G-7) and crop lavage (G-3) was taken from four chickens from each group that were euthanized on different period of interval: day 0, 21, 42, and 56 of the experiment; thus, a total of 16 chickens from each group were euthanized for this purpose. Trachea and crop were washed by glycine buffer and analyzed for the presence of IgA. The data were presented as individual values for each experimental group. Mean and standard error of means are indicated in lines and error bars. Asterisk (*) represents the significant differentiation of antibody lgA level compared to the non-vaccinated control group ( $\left.{ }^{*} \mathrm{p}<0.05\right)$.

administered intranasally. Complete protection of the chickens from fowl cholera was conferred by vaccination with the intranasal route of gamma-irradiated fowl cholera vaccine or by intraocular route of gamma-irradiated fowl cholera $(0.3 \mathrm{ml} /$ dose $)$ vaccine. The protective efficacy in the chickens immunized with the gammairradiated oral vaccine and formalin-inactivated $\mathrm{FC}$ vaccine was 85 and $80 \%$, respectively, while in those immunized with the gammairradiated intraocular $(0.5 \mathrm{ml})$ vaccine was $90 \%$. According to the log-rank test for equality survival function, vaccinated groups in both vaccine types showed significant difference compared to the control group $(\mathrm{p}<0.001)$. Furthermore, there was significant difference between the protection conferred by the gammairradiated mucosal fowl cholera vaccine and formalin-inactivated FC vaccine $(\mathrm{p}<0.001)$. In addition, the survival rate of chickens vaccinated with gamma-irradiated FC vaccine intranasally was significantly different compared to chickens vaccinated through the oral route $(\mathrm{p}<0.001)$.

Based on the curve, there was not any chicken that survived after exposure to the challenge bacterial strain in the control group. The death of chickens started 2 days after challenge, and all of the chickens in this group died within 7 days. In addition, three and four chickens in gamma-irradiated mucosal FC vaccine through oral route and formalin-inactivated immunized groups, respectively, and two chickens in the gamma-irradiated mucosal FC vaccine through intraocular route $(0.5 \mathrm{ml} /$ dose $)$ immunized group died within 10 days, respectively (Figure 6).

\section{Clinical Signs, Gross Lesions, and Bacterial Isolation}

After challenge, no behavioral changes were detected in the groups of chickens immunized with gamma-irradiated mucosal FC vaccine through intranasal and intraocular $(0.3 \mathrm{ml} / \mathrm{dose})$ routes. In the control groups, chickens manifested clinical signs at $24 \mathrm{~h}$ after the challenge exposure, including depression, anorexia, and severe diarrhea. During the period of 4 days, the severity increased rapidly, resulting in the death of several chickens, and all of the chickens in this group died within 7 days. In addition, seven and eight chickens in the gamma-irradiated mucosal FC vaccine through oral route and formalin-inactivated immunized groups, respectively, and five chickens in the gamma-irradiated mucosal FC vaccine through intraocular route $(0.5 \mathrm{ml} /$ dose $)$ immunized group started to display depression and anorexia 5 days after the challenge exposure. Among these chicken, three, four, and two chickens then died within 10 days, respectively.

All of the dead chickens in this investigation had characteristic lesions of fowl cholera, including lung congestion, lung edema, and numerous petechiae in the liver, hemorrhage in the small intestine, splenomegaly, and fibrinopurulent peritonitis, according to necropsy results (Figure 7). In addition, the dead chickens were also subjected to isolation and identification of avian $P$. multocida. The findings revealed that avian $P$. multocida was recovered in pure cultures from all of the dead chicken specimens, which was furthermore confirmed by species-specific PCR.

\section{DISCUSSION}

Fowl cholera caused by $P$. multocida is a highly contagious disease of poultry presenting as one of the major challenges worldwide. It affects the poultry industry, incurring economic losses due to loss of products (19). The development of vaccine to control FC has proven to be a challenge for years. An effective vaccine must be safe and needs to provide sustained protection with elimination of the challenge infection. Live and formalininactivated FC vaccines have been extensively used and succeeded in reducing infection and the prevalence of disease in poultry but have limitations associated with safety and elicit protections of short duration (20). In addition, parenteral vaccines have limited ability of inducing mucosal immunity, which is key in protection against infection or disease by mucosal pathogens. Furthermore, parenterally administered vaccines are stressful to birds and not suitable for mass vaccination, requiring much labor and time. Currently, there is a need for mucosal 


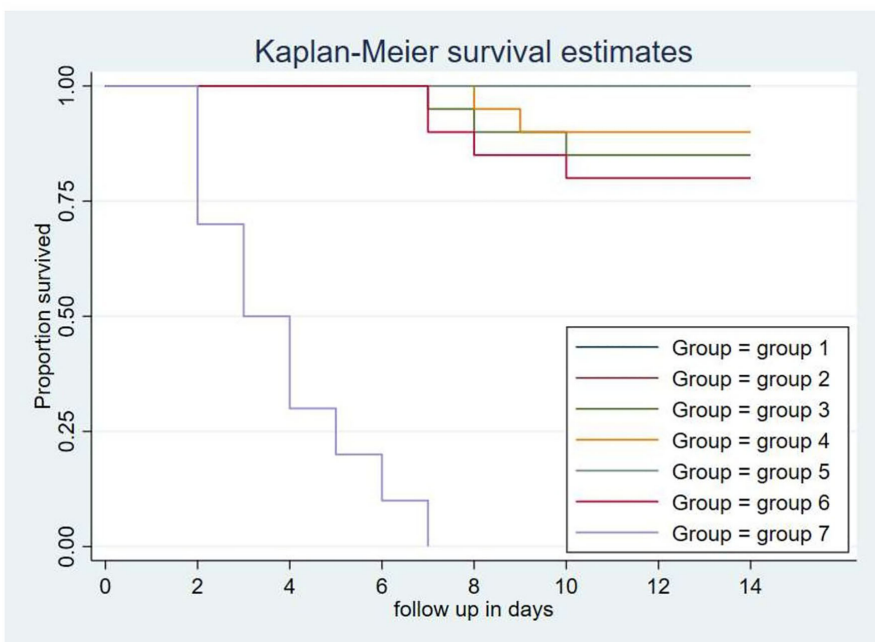

FIGURE 6 | Each group comprises 20 chickens that had been followed for the period of 14 days. The chickens in the treatment and control groups were given 0.5 $\mathrm{ml}$ of avian P. multocida biotype A. The data were used to determine the Kaplan-Meier estimates (the product limit estimate) of both the control and the treatment groups. The curve takes a step down when the chickens were dead.

vaccines against pathogens that invade via the mucosal surfaces. This route of vaccine delivery would also eliminate needle injections (7).

The present study is aimed to develop and evaluate gammairradiated mucosal FC vaccine that can be utilized nationally to curb the impact of the disease in Ethiopia as well as in other African countries. In a preliminary study we conducted, fresh cultures of avian P. multocida $\left(5.6 \times 10^{9} \mathrm{CFU} / \mathrm{ml}\right)$ were irradiated with different doses $(0.9,1,1.1$, and $1.2 \mathrm{kGy})$ of gamma radiation.
Then, the safety and immunogenicity of these preparations [after addition of Montanide gel/01 PR adjuvant (18)] were evaluated in chickens to select the superior radiation dose. Accordingly, irradiation with $1 \mathrm{kGy}$ resulted in safe and immunogenic preparation as evidenced by the higher titer of antibody elicited in chickens and its safety.

Gamma irradiation has been used extensively as an alternative inactivation method of pathogens because of its high penetrability, which allows bacterial inactivation in large
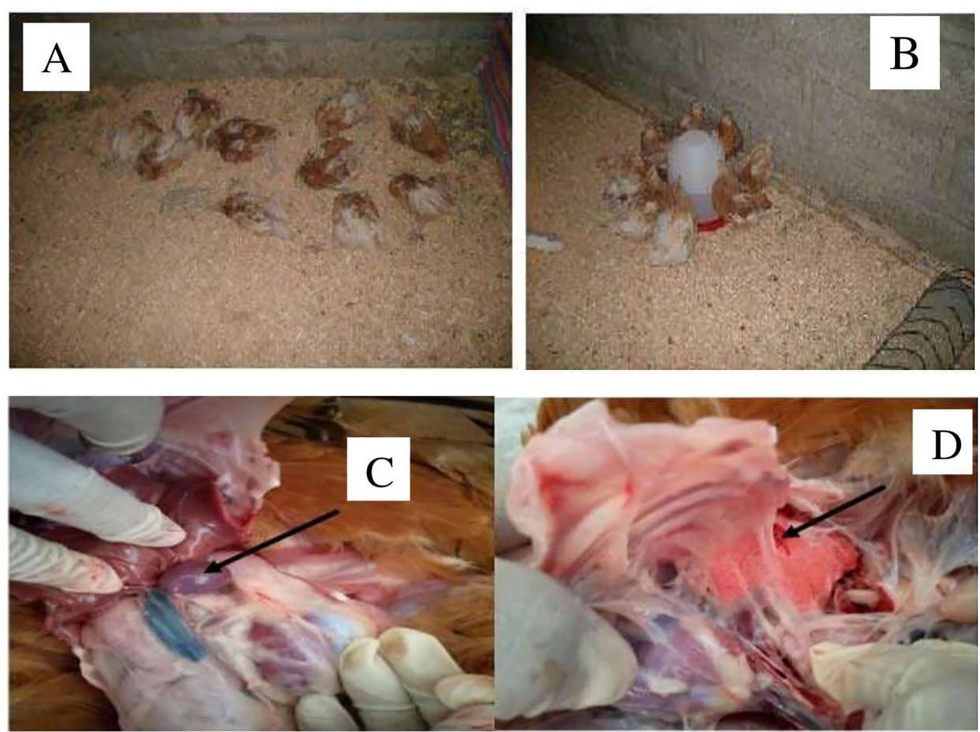

FIGURE 7 | The picture from (A-D) indicated the chickens died after challenge with P. multocida biotype A at day 56 and the postmortem result. (A, B) indicated the dead chickens within 2 weeks. (C, D) indicated splenomegaly and petechial and congested lung. 
volumes within a short time via damage of nucleic acid (11). Several previous reports have suggested that nucleic acids rather than proteins are the primary targets of gamma irradiation to inactivate microorganisms. For example, gamma-irradiated $S$. pneumoniae vaccine produced after inactivation with $10 \mathrm{kGy}$ gamma irradiation elicits strong mucosal and systemic immune responses in mice model, which is indicative of the affectivity of gamma irradiation as a method for the development of a killed whole-cell pneumococcal vaccine (21). Furthermore, sterilization doses for radiation-sensitive organisms could be significantly reduced, which would be expected to reduce damage to epitopes required to develop a protective immune response while maintaining an adequate margin of safety to ensure complete inactivation (22).

In our study, a dose range of 1-3 kGy completely inactivated the avian $P$. multocida as confirmed by the subculturing on TSA and TSB while retaining its immunological properties. There are no available reports on inactivation of avian $P$. multocida using gamma rays. However, $M$. haemolytica was reported to be successfully irradiated using gamma rays of $20 \mathrm{kGy}$ doses, a dose selected to be optimal for vaccine preparation (23). According to our study, radiation with $1 \mathrm{kGy}$ resulted in no avian $P$. multocida cell survival, and the resultant vaccine formulation induced significantly higher antibody response than the formalin-inactivated vaccine. This implies that gamma irradiation efficiently inactivates bacteria with less impact on antigenic structures (determinants), leading to a robust immune response. In contrast, formalin inactivation has been known to induce the formation of methylene bridges between amino groups, resulting in protein cross-linking, affecting antigenicity (24).

One of the key determinants of effectiveness of vaccines is the adjuvant selection. Montanide gel/01 PR is an innovative polymeric adjuvant designed to improve the safety and efficacy of aqueous vaccines. Those adjuvants are based on a dispersion of highly stable gel particles of sodium polyacrylate in water (25). The depot effect with slow release due to polymer adsorption properties improves the recruitment of the innate immune system. It provides a significant enhancement of systemic and mucosal immune responses with a better safety performance than potassium aluminum sulfate (Alum) (26). Based on our finding, it can be speculated that the presence of Montanide gel in our vaccine formulation contributed for its better immunogenicity and efficacy as compared to formalin-inactivated FC vaccine. However, this requires further investigation.

Avian P. multocida is known to cause disease in poultry species by infecting or entering through the mucosal surface of the upper respiratory tract. Thus, the first line of defense of the host is invoked against inhaled antigens, making the respiratory route potentially the most effective route for vaccination that is capable of inducing both systemic and mucosal immunity (8). Mucosal vaccines administered through IN route mimics the route of natural infection of mucosal pathogens such as avian $P$. multocida, which in turn would result in protective immune response than injectable preparations (6).
The gamma-irradiated mucosal FC vaccine developed in this study was evaluated for its ability to induce both serum IgG and mucosal IgA in chickens. In chicken sera, IgG is the most common immunoglobulin form, and secretory IgA is produced locally by plasma cells located at mucosal surfaces and plays an important role in mucosal immunity (27). This finding indicated that antibody titers in sera of chickens vaccinated with gamma-irradiated mucosal vaccine were significantly increased after 2 weeks post-vaccination, but significant shooting was recorded at 6 and 8 weeks post-vaccination and is in agreement with (8) and (28), who stated that rOmpHLTB-based intranasal and irradiated bacterial vaccines generate higher humoral immune responses and protection against extracellular and intracellular bacteria and (29) who registered that vaccines developed by irradiation have been found to be strong inducers for humoral immune responses that make this type of vaccine highly effective.

An interesting finding in our study was the gamma-irradiated mucosal fowl cholera vaccine led to high levels of $P$. multocidaspecific serum IgG responses as compared to formalininactivated fowl cholera vaccine. This might be due to highly preserved immunogenic properties of protein antigens even after irradiation. However, formalin inactivation can cause crosslinking of several amino acid residues, which leads to a lower immunogenic response (30).

In regard to efficacy, our finding showed that vaccination with intranasal and intraocular $(0.3 \mathrm{ml} /$ dose $)$ route of the gammairradiated mucosal vaccine resulted in $100 \%$ protection against lethal challenge. As compared to the intranasal route, immunization through oral and intraocular route resulted in less efficacy $(p<0.05)$ as shown by death of some birds in those groups. This can be due to the local IgA produced in the mucosal airways, which is the natural route and of infection of FC (21).

In this study, the gamma-irradiated fowl cholera vaccine was safe as chickens injected with it were devoid of vaccination side effects and their bodies were maintained normal, and it avoids the drawbacks in vaccinated chickens with chemical inactivated fowl cholera vaccines. We believe that these preliminary findings demonstrate that the gammairradiated mucosal fowl cholera vaccine approach is an adaptable vaccine strategy against avian $P$. multocida and that this information will aid in the evaluation of other whole-cell, killed vaccine strategies, as well as identify candidates for recombinant protein vaccine approaches.

In conclusion, the present study showed that gammairradiated FC mucosal vaccine is safe and protective, suggesting its potential use for immunization of chicken against FC in chicken. One kGy was identified as a dosage of gamma irradiation that inactivated $P$. multocida replication while retaining immunogenic surface structures. Montanide gel/01 PR showed a significant enhancement of systemic and mucosal immune responses with a safety. In this study, the candidate gamma-irradiated mucosal vaccines induced higher response of both serum IgG and mucosal IgA after three IN doses, the latter (IgA) being highly relevant in the context of protective immunity. In addition to its good immunogenicity, 
the candidate vaccine provided protection in challenge experiments. This can be considered a go-on signal to further evaluate the vaccine and approve it for national use.

\section{DATA AVAILABILITY STATEMENT}

The original contributions presented in the study are included in the article/supplementary material. Further inquiries can be directed to the corresponding author.

\section{ETHICS STATEMENT}

The animal study was reviewed and approved by the animal research ethics committee of the National Veterinary Institute, Ethiopia with the protocol number NVI-AE-008-2020.

\section{AUTHOR CONTRIBUTIONS}

$\mathrm{BD}$ and $\mathrm{EK}$ were involved in the data collection. $\mathrm{BD}, \mathrm{DA}$, and $\mathrm{SI}$ participated in the study design and in the laboratory analysis, performed data analysis, and drafted the manuscript. MB, EG, TA, VW, and HU assisted in study conception and manuscript revision and also assisted in analysis interpretation and gave inputs in the final manuscript. EG, TA, VW, and HU read and

\section{REFERENCES}

1. Asfaw YT, Yohannes T, Ameni G, Medhin G, Gumi B, Hagos Y, et al. Poultry Disease Occurrences and Their Impacts in Ethiopia. Trop Anim Health Production (2021) 53(1):54. doi: 10.1007/s11250-020-02465-6

2. Molalegne B, Kelay B, Berhe GE, Kyule M. Development and Efficacy Trial of Inactivated Fowl Cholera Vaccine Using Local Isolates of Pasteurellamultocida. Ethiopian Veterinary J (2009) 13(2):81-98.

3. World organization for animal health. Manual of Diagnostic Tests and Vaccines for Terrestrial Animals. Paris, France (2019). Access on: https:// www.oie.int/en/produit/manual-of-diagnostic-tests-and-vaccines-forterrestrial-animals-2019/.

4. Fan YC, Chiu HC, Chen LK, Chang GJ, Chiou SS. Formalin Inactivation of Japanese Encephalitis Virus Vaccine Alters the Antigenicity and Immunogenicity of a Neutralization Epitope in Envelopeproteindomainiii. PLoSNegl.Trop.Dis. (2015) 9:10. doi: 10.1371/journal.pntd.0004167

5. Lycke N. Recent Progress in Mucosal Vaccine Development: Potential and Limitations. Nat Rev Immunol (2017) 12:592. doi: 10.1038/emm.2014.2

6. Miquel-Clopes A, Bentley EG, Stewart JP, Carding SR. Mucosal Vaccines and Technology. Clin Exp Immunol (2019) 196:205-14. doi: 10.1111/cei.13285

7. Thanasarasakulpong A, Poolperm P, Tankaew P, Sawada T, Sthitmatee N. Protectivity Conferred by Immunization With Intranasal Recombinant Outer Membrane Protein H From Pasteurella Multocida Serovar a:1 in Chickens. J Veterinary Med Sci (2015) 77(3):321-6. doi: 10.1292/jvms.14-0532

8. Poolperm P, Apinda N, Kataoka Y, Suriyasathaporn W, Tragoolpua K, Sawada T, et al. Protection Against Pasteurellamultocida Conferred by an Intranasal Fowl Cholera Vaccine in Khaki Campbell Ducks. Japanese J Veterinary Res (2018) 66(4):239-50. doi: 10.14943/jjvr.66.4.239

9. Woodrow KA, Bennett KM, Lo DD. Mucosal Vaccine Design and Delivery. Annu Rev Biomed.Eng (2012) 14:17-46. doi: 10.1517/17425247.2013.740008

10. Seo HS. Application of Radiation Technology in Vaccines Development. Clin Exp Vaccine Res (2015) 4(2):145. doi: 10.7774/cevr.2015.4.2.145

11. Fertey J, Bayer L, Kahl S, Rukiya M, Burger-Kentischer A, Thoma M, et al. Low-Energy Electron Irradiation Efficiently Inactivates the Gram-Negative Pathogen Rodentibacterpneumotropicus New Method for the Generation of commented on the manuscript and rearranged for publication. All authors contributed to the article and approved the submitted version.

\section{FUNDING}

This work was supported by the CRP grant (code D32035) from the International Atomic Energy Agency (IAEA), Vienna, Austria.

\section{ACKNOWLEDGMENTS}

The study was supported by a CRP grant from the International Atomic Energy Agency (IAEA). The authors are very grateful for the support provided from Dr Giovanni Cattoli and Dr RichardKangethe of IAEA. Special thanks go to Sebastien Deville of SEPPIC, Air Liquide Healthcare Specialty Ingredients for kindly providing us Montanide/01 PR gel. The authors would like tothank the National Veterinary Institute (NVI) of Ethiopia for availing the laboratory and animal experimentation facilities. The technical support of the staff at the Research and Development directorate of NVI is also highly appreciated. We also thank National Institute for Control and Eradication of Tsetse Fly and Trypanosomosis of Ethiopia for allowing us to use the irradiation facility.

Bacterial Vaccines With Increased Efficacy. Vaccines (2020) 8:1-9. doi: 10.3390/vaccines8010113

12. Bayer L, Fertey J, Ulbert S, Grunwald T. Immunization With an Adjuvanted Low-Energy Electron Irradiation Inactivated Respiratory Syncytial Virus Vaccine Shows Immune Protective Activity in Mice. Vaccine (2018) 36:1561-9. doi: 10.1016/j.vaccine.2018.02.014

13. Syaifudin M, Tetriana D, Nurhayati S. The Feasibility of Gamma Irradiation for Developingmalariavaccine. AtomIndonesia (2011) 37(3):91-101. doi: 10.13170/ ajas.4.1.13558

14. National Veterinary institute/SOP. Fowl Cholera Vaccine Production NVI Standard Operational Procedure. Debre zeit, Ethiopia (2017) 1-7.

15. Begum RH, Rahman H, Ahmed G. Development and Evaluation of Gamma Irradiated Toxoid Vaccine of Salmonella Entericavartyphimurium. Veterinary Microbiol (2011) 153(1-2):191-7. doi: 10.1016/j.vetmic.2011. 06.013. Elsevier B.V.

16. Ahmad TA, Rammah SS, Sheweita SA, Haroun M, El-Sayed LH. Development of Immunization Trials Against Pasteurellamultocida. Vaccine (2014) 32:90917. doi: 10.1016/j.vaccine.2013.11.068

17. Gaidamakova EK, Elena K, Myles A, McDaniel DP, Fowler CJ, Valdez P, et al. Preserving Immunogenicity of Lethally Irradiated Viral and Bacterial Vaccine Epitopes Using a Radio-Protective Mn2+-Peptide Complex From Deinococcus. Cell Host Microbe (2012) 12(1):117-24. doi: 10.1016/j.chom.2012.05.011

18. Seppic. Technical Bulletin, Montanide TM01 Gel Ready to Use Aqueous Polymeric Adjuvant for Veterinary Vaccines (2021). Available at: WWW. Seppic.Comhttps://www.seppic.com/sites/seppic/files/2017/02/28/seppicmontanide.pdf.

19. Wubet W, Bitew M, Mamo G, Gelaye E, Tesfaw L, Sori H, et al. Evaluation of Inactivated Vaccine Against Fowl Cholera Developed From Local Isolates of Pasteurellamultocida in Ethiopia. Afr J Microbiol Res (2019) 13:500-9. doi: 10.5897/AJMR2019.9096

20. Harper M, Boyce D. The Myriad Properties of Pasteurellamultocida Lipopolysaccharide. Toxin (2017) 9:254. doi: 10.3390/toxins9080254

21. Jwa MY, Jeong S, Ko EB, Kim R, Seo HS, Yun C, et al. Gamma-Irradiation of Streptococcus Pneumoniae for the Use as an Immunogenic Whole Cell Vaccine. J Microbiol (2018) 56(8):579-85. doi: 10.1016/j.micpath.2018.08.015 
22. Dollery J, Zurawski V, Gaidamakova E, Matrosova V, Tobin J, Wiggins T, et al. Radiation-Inactivated Acinetobacterbaumanniivaccine Candidates. Vaccines (2021) 9(2):1-16. doi: 10.3390/vaccines 9020096

23. Sahar A, Basem S, Ahmed G, Mahmoud I, Waleed N, Abdel-Rahim EA. Development of Gamma Irradiation Vaccine Against Mannheimiahaemolytica. Res J Immunol (2015) 8(1):17-26. doi: 10.3923/rji.2015.17.26

24. Thavarajah R, Mudimbaimannar VK, Elizabeth J, Rao UK, Ranganathan K. Chemical and Physical Basics of Routine Formaldehyde Fixation. J Oral MaxillofacPathol (2012) 16:400-5. doi: 10.4103/0973-029X.102496

25. Damiana T, Portuondo D, Loesch M, Batista-Duharte A, CarlosI IZ. A Recombinant Enolase-Montanide TM Petgel a Vaccine Promotes a Protective Th1 Immune Response Against a Highly Virulent Sporothrixschenckii by Toluene Exposure. Pharmaceutics (2019) 11:144. doi: 10.3390/pharmaceutics11030144

26. Jafari M, Moghaddam M, Taghizadeh M, Masoudi S, Bayat Z. Comparative Assessment of Humoral Immune Responses of Aluminum Hydroxide and Oil-Emulsion Adjuvants in Influenza (H9N2) and Newcastle Inactive Vaccines to Chickens. Artif Cells Nanomed Biotechnol (2017) 45:84-9. doi: 10.3109/21691401.2015.1129626

27. Varinrak T, Poolperm P, Sawada T, Sthitmatee N. Cross-Protection Conferred by Immunization With an Romph-Based Intranasal Fowl Cholera Vaccine. Avian Patho (2017) 46:515-25. doi: 10.1080/03079457.2017.1321105

28. Tuasikal BJ, Wibawan F, Pasaribu H, Estuningsih S. Bacterial Protein Characterization of Streptococcus Agalactiae by SDS-PAGE Method for Subclinical Mastitis Irradiated Vaccine Materials in Dairy Cattle. Atom Indonesia (2012) 38:66-70. doi: 10.17146/aij.2012.162
29. Mahmoud HI, Makharita MA, Abbas N. Comparison Between Protective Immunity Induced by Gamma-Irradiated Brucellaabortus Field Strain and Commercial Brucellaabortus Strain 19 in Mice. Intl J Microbiol Res (2016) 7 (3):114-9. doi: 10.5829/idosi.ijmr.2016.114.119

30. Babb R, Chen A, Hirst TR, Kara EE, McColl SR, Ogunniyi AD, et al. Intranasal Vaccination With-Irradiated Streptococcus Pneumoniae Whole-Cell Vaccine Provides Serotype-Independent Protection Mediated by B-Cells and Innate IL-17 Responses. Clin Sci (2016) 130(9):697-710. doi: 10.1042/CS20150699

Conflict of Interest: The authors declare that the research was conducted in the absence of any commercial or financial relationships that could be construed as a potential conflict of interest.

Publisher's Note: All claims expressed in this article are solely those of the authors and do not necessarily represent those of their affiliated organizations, or those of the publisher, the editors and the reviewers. Any product that may be evaluated in this article, or claim that may be made by its manufacturer, is not guaranteed or endorsed by the publisher.

Copyright (C) 2021 Dessalegn, Bitew, Asfaw, Khojaly, Ibrahim, Abayneh, Gelaye, Unger and Wijewardana. This is an open-access article distributed under the terms of the Creative Commons Attribution License (CC BY). The use, distribution or reproduction in other forums is permitted, provided the original author(s) and the copyright owner(s) are credited and that the original publication in this journal is cited, in accordance with accepted academic practice. No use, distribution or reproduction is permitted which does not comply with these terms. 